\title{
Enfermagem Psiquiátrica, Saúde Mental e as Diretrizes Curriculares Nacionais do Curso de Graduação em Enfermagem*
}

Amanda Márcia dos Santos Reinaldo ${ }^{1}$

(D) https://orcid.org/0000-0003-0283-2313

Girliani Silva de Sousa ${ }^{2}$

(D) https://orcid.org/0000-0002-0988-5744

Belisa Vieira da Silveira ${ }^{3,4}$

(D) https://orcid.org/0000-0002-5966-8537
* A publicação deste artigo na Série Temática "Recursos Humanos em Saúde e Enfermagem" se insere na atividade 2.2 do Termo de Referência 2 do Plano de Trabalho do Centro Colaborador da OPAS/OMS para o Desenvolvimento da Pesquisa em Enfermagem, Brasil.

${ }_{1}^{1}$ Universidade Federal de Minas Gerais, Departamento de Enfermagem Aplicada, Belo Horizonte, MG, Brasil.

2 Universidade Federal de São Paulo, Escola de Enfermagem. Departamento de Enfermagem Clínica-Cirúrgica, São Paulo, $\mathrm{SP}$, Brasil.

${ }^{3}$ Universidade de São Paulo, Escola de Enfermagem de Ribeirão Preto, Centro Colaborador da OPAS/OMS para o Desenvolvimento da Pesquisa em Enfermagem, Ribeirão Preto, SP, Brasil.

${ }^{4}$ Bolsista da Coordenação de Aperfeiçoamento de Pessoal de Nível Superior (CAPES), Brasil.
Objetivo: analisar as recomendações para a reformulação das Diretrizes Curriculares Nacionais do Curso de Graduação em Enfermagem (Resolução no 573, de 31 de janeiro de 2018) no que tange ao ensino da Enfermagem Psiquiátrica e Saúde Mental no processo formador do enfermeiro. Método: trata-se de um estudo documental. A coleta de dados foi realizada a partir de busca textual sobre o tema em diferentes sítios de pesquisa. Os dados foram tabulados com o uso do software Web Qualitative Data Analysis e submetidos à Análise de Conteúdo. As categorias encontradas foram: O cuidado de Enfermagem em saúde mental é um direito de todos; O cuidado de Enfermagem em saúde mental considera a singularidade do sujeito. Resultados: as recomendações fortalecem o ensino de Enfermagem Psiquiátrica e Saúde Mental, trazendo protagonismo para a área no contexto do cuidado em saúde. Conclusão: as recomendações atendem às questões contemporâneas associadas ao sofrimento mental nas populações. A formação do enfermeiro deve atender às questões sociais, políticas e do mercado, considerando os determinantes sociais em saúde e saúde mental.

Descritores: Programas de Graduação em Enfermagem; Enfermagem Psiquiátrica; Currículo; Educação em Enfermagem.

\section{Como citar este artigo}

Reinaldo AMS, Souza GSS, Silveira BV. Psychiatric Nursing, Mental Health and the National Curriculum Guidelines for the Undergraduate Nursing Course. SMAD, Rev Eletrônica Saúde Mental Álcool Drog. 2021 jul.-set.;17(3):57-66. doi: https://dx.doi.org/10.11606/issn.1806-6976.smad.2021.174632 


\title{
Psychiatric Nursing, Mental Health and the National Curriculum Guidelines for the Undergraduate Nursing Course
}

\begin{abstract}
Objective: to analyze the recommendations for the reformulation of the National Curricular Guidelines of the Undergraduate Course in Nursing (Resolution No. 573 of January 31, 2018) regarding the teaching of Psychiatric Nursing and Mental Health in the formative process of nurses. Method: this is a documental study. Data was collected from a textual search on the theme in different research sites. The data were tabulated using Web Qualitative Data Analysis software and submitted to Content Analysis. The categories found were Nursing care in mental health is a right for all; Nursing care in mental health considers the singularity of the subject. Results: the recommendations strengthen the teaching of Psychiatric and Mental Health Nursing, bringing protagonism to the area in the context of health care. Conclusion: the recommendations address contemporary issues associated with mental suffering in populations. Nursing education should address social, political and market issues, considering the social determinants in health and mental health.
\end{abstract}

Descriptors: Graduate Programs in Nursing; Psychiatric Nursing; Curriculum; Nursing Education.

\section{Enfermería psiquiátrica, salud mental y las directrices curriculares nacionales para el curso de pregrado en enfermería}

\begin{abstract}
Objetivo: analizar las recomendaciones para la reformulación de los Lineamientos Curriculares Nacionales de la carrera de pregrado en (Resolución no 573 del 31 de enero de 2018) sobre la docencia de Enfermería Psiquiátrica y Salud Mental en el proceso de formación del enfermero. Método: se trata de un estudio documental. La recolección de datos se realizó a partir de una búsqueda textual sobre el tema en diferentes sitios de investigación. Los datos se tabularon utilizando el software Web Qualitative Data Analysis y se sometieron a análisis de contenido. Las categorías encontradas fueron: La atención de enfermería en salud mental es un derecho de todos; El cuidado de enfermería en salud mental considera la singularidad del sujeto. Resultados: las recomendaciones fortalecen la enseñanza de la enfermería psiquiátrica y en salud mental, otorgando protagonismo al área en el contexto de la atención de la salud. Conclusión: las recomendaciones abordan problemas contemporáneos asociados con el sufrimiento mental en las poblaciones. La formación de enfermeras debe atender cuestiones sociales, políticas y de mercado considerando los determinantes sociales en salud y salud mental.
\end{abstract}

Descriptores: Programas de Posgrado en Enfermería; Enfermería Psiquiátrica; Curriculum; Educación en Enfermería. 


\section{Introdução}

No ano internacional dedicado à Enfermagem, uma pandemia por COVID-19 evidenciou o trabalho essencial da equipe de Enfermagem nos sistemas de saúde na linha de cuidado ao rastreamento das pessoas contaminadas, tratamento ofertado aos casos leves e graves de pessoas infectadas por COVID-19 e na assistência às pessoas em sofrimento psíquico.

O isolamento social necessário e por tempo indeterminado de milhões de pessoas no mundo contribuiu para evidenciar a fragilidade da população em relação às questões relacionadas à saúde mental, sua promoção, proteção e cuidado. Enfermeiros na linha de frente assistencial e na retaguarda dos serviços buscaram e ofereceram suporte psicossocial para fazer frente a uma situação grave de saúde com desdobramentos que não podem ser mensurados em curto prazo(1-4).

Essa realidade chama a atenção em nível mundial para a necessidade de educação de qualidade para o desenvolvimento de competências profissionais da Enfermagem frente à oferta de cuidado a uma pandemia ocasionada por um vírus ainda de causas e efeitos desconhecidos nos indivíduos(5-7).

A Enfermagem, nesse sentido, viu-se diante de desafios em diferentes frentes de trabalho que prescindiam de seu conhecimento e da qualidade de sua formação. Nesse contexto, a formação do enfermeiro no mundo acompanhou a história da saúde, as políticas públicas e os movimentos sociais que modificaram, criaram e consolidaram os sistemas de saúde, tais como, no caso do Brasil, a Reforma Sanitária, a Reforma Psiquiátrica e a criação do Sistema Único de Saúde (SUS) ${ }^{(8-12)}$.

A elaboração das Diretrizes Nacionais Curriculares do Curso de Graduação em Enfermagem, em 2001, configura-se como estratégia potente para o direcionamento da formação de enfermeiros no SUS e para o SUS. Em seu escopo, as DCNs traçaram o perfil do profissional adequado e possível na época de sua elaboração; também definiram as competências gerais e específicas que deveriam orientar os projetos políticos pedagógicos e os currículos das escolas de Enfermagem no Brasil(13).

A publicação das DCNs trouxe avanços e direcionamento para a formação, embora, para alguns autores, tenham se distanciado do conhecimento das ciências humanas, deixando em segundo plano a formação humanística do enfermeiro(8,14-17).

As DCNs são a referência para a elaboração dos Projetos Políticos Pedagógicos e Currículos de Enfermagem. Esses documentos são revisados de acordo com as diretrizes, expandindo as ações dos enfermeiros e formando uma estrutura para o ensino da Enfermagem em sintonia com o mercado de trabalho, as boas práticas de saúde baseadas em evidências, o perfil epidemiológico das populações, seus determinantes e condicionantes sociais e econômicos em saúde.

No Brasil, os desafios para a qualidade na formação de enfermeiros generalistas envolvem: a divisão social do trabalho; o modelo positivista de cuidado em saúde; os currículos organizados em disciplinas; a expansão do número de escolas de Enfermagem no país; a dificuldade de avaliar a qualidade de ensino dessas instituições e a discussão em torno do ensino na modalidade Educação a Distância (EAD), que podem fragilizar a profissão enquanto um campo do saber em saúde(18-21).

A formação generalista do enfermeiro está embasada em competências e habilidades gerais e específicas que sustentam suas práticas em diferentes pontos de atenção(22-24).

Em 2018, a Resolução no 573, de 31 de janeiro, aprovou o Parecer Técnico no 28/2018 contendo recomendações do Conselho Nacional de Saúde (CNS) à proposta de DCNs para os cursos de graduação em Enfermagem(17).

Observa-se, em relação ao ensino da Enfermagem Psiquiátrica e Saúde Mental, um avanço entre as DCNs (2001) e a proposta aprovada na Resolução no 573, de 2018. No documento de 2001, não há menção textual a questões do cuidado em Enfermagem Psiquiátrica e Saúde Mental nas 33 competências da Enfermagem descritas e no seu texto em geral, carecendo de exatidão textual no que se refere a uma área importante do cuidado integral em saúde e que faz interface com outras áreas, entre elas, a área de álcool e outras drogas. Identifica-se, portanto, um caráter contemporâneo na resolução no que se refere às necessidades de saúde da população em relação ao sofrimento mental.

A necessidade de o enfermeiro generalista desenvolver competências para o cuidado em Enfermagem Psiquiátrica e Saúde Mental, nos diferentes contextos assistenciais, é crucial diante do panorama contemporâneo advindo da pandemia por COVID-19 com o aumento dos casos de depressão, distúrbios do sono, transtornos de ansiedade, risco de suicídio, uso de álcool e outras drogas, estresse, violência doméstica e causas associadas na população geral.

Ressalta-se que tais competências são necessárias para o estabelecimento de estratégias para dar suporte à população e aos trabalhadores de saúde como: o atendimento on-line; a produção de materiais informativos; as iniciativas de organizações ligadas à cultura e ao lazer; os protocolos de atendimento; as recomendações a serem elaboradas e disponibilizadas com o intuito de ajudar no manejo da incerteza, medo, isolamento e solidão(1-2,25). No Brasil, tais atividades ainda são incipientes frente às demandas sociais, econômicas e psicológicas das pessoas.

Nesse sentido, este estudo teve por objetivo analisar as recomendações para a reformulação das DCNs do curso de graduação em Enfermagem (Resolução no 573, de 31 de janeiro de 2018) no que tange ao ensino da 
Enfermagem Psiquiátrica e Saúde Mental no processo formador do enfermeiro generalista.

\section{Método}

Trata-se de um estudo de análise documental. As fontes primárias de análise foram a Resolução no 573, de 31 de janeiro de 2018, que versa sobre as recomendações para a reformulação das DCNs do curso de graduação em Enfermagem; as DCNs de Enfermagem (2001); a Lei no 10.216, de 6 de abril de 2001, que dispõe sobre a proteção e os direitos das pessoas portadoras de transtornos mentais e redireciona o modelo assistencial em saúde mental e o Relatório Mundial da Saúde 2001 "Saúde mental: nova concepção, nova esperança"(26).

A pesquisa compreendeu a identificação, a verificação e a análise de documentos que mantêm relação com o objeto investigado, permitindo a discussão teórica do tema em questão. Os documentos foram submetidos à Análise de Conteúdo, sistematizada por Bardin, e compreendeu: (1) Pré-análise; (2) Exploração do material e (3) Tratamento dos resultados(27-28). A coleta de dados foi realizada no mês de maio de 2020 a partir de busca textual sobre o tema na web em diferentes sítios de pesquisa de livre acesso.

Outros estudos e documentos foram utilizados como fonte secundária de informação, entre eles, artigos científicos, relatórios, informes de organizações de saúde e resoluções sobre o exercício profissional do enfermeiro expedidas pelo Conselho Federal de Enfermagem (COFEN).

Os dados foram tabulados com o uso do software de análise de textos, vídeos, áudios e imagens Web Qualitative Data Analysis (WebQDA). O sistema é organizado em três áreas: 1 . Fontes - onde o sistema é alimentado com os dados da pesquisa e organizados de acordo com a necessidade do investigador; 2. Criação de codificação ou categorias e 3. Questionamento - o investigador cria as dimensões e/ou categorias que serão analisadas de acordo com modelos de análise previamente elaborados para cada uma delas(29).

Dessa forma, os documentos foram anexados à plataforma integralmente (Fonte) e foram definidas palavras-chaves em relação ao tema para que o sistema as identificasse nos textos, a saber: saúde mental; Enfermagem; Enfermagem Psiquiátrica; álcool; drogas; ensino; avaliação; cuidado; promoção à saúde; prevenção; tratamento; assistência integral; integralidade; determinantes sociais em saúde; pessoa; sujeito; paciente e comunidade (codificação). Por fim, foram acrescentadas as categorias prévias de acordo com um modelo de análise criado a partir da leitura inicial dos documentos.

As categorias elencadas foram: (1) O cuidado de Enfermagem em saúde mental é um direito de todos; (2) O cuidado de Enfermagem em saúde mental considera a singularidade do sujeito.
O artigo é um recorte de um estudo maior intitulado "Estudo e Pesquisa em Boas práticas em Enfermagem Psiquiátrica e Saúde Mental", que aborda o sofrimento mental como algo intrinsecamente ligado aos condicionantes e determinantes sociais de saúde e saúde mental, associado à formação do enfermeiro generalista por meio do cuidado primário em saúde mental, das tecnologias do cuidado e da validação das competências necessárias para o enfermeiro generalista atuar na atenção à pessoa em sofrimento em diferentes cenários.

\section{Resultados e Discussão}

O cuidado de Enfermagem em saúde mental é um direito de todos

O cuidado de Enfermagem é um direito de todos, asseguram as DCNs de Enfermagem(30). A Resolução no 573, de 31 de janeiro de $2018^{(17)}$, corrobora esse direito. Nesse sentido, as recomendações do Conselho Nacional de Saúde (CNS) à proposta das diretrizes trazem avanços para a formação do egresso ao incorporar textualmente a necessidade do profissional em formação, atendendo às necessidades individuais e coletivas, e respeitando as diversidades mentais, a pluralidade humana, a singularidade do ser humano e os princípios básicos da ação terapêutica, entre eles, as condições biopsicossociais.

A Reforma Psiquiátrica tem como marco legal a Lei no 10.216, de 6 de abril de 2001, que garantiu o direito da pessoa em sofrimento mental à informação sobre o transtorno mental e seu tratamento, o acesso ao tratamento de qualidade em serviços comunitários de saúde mental de acordo com as suas necessidades e a autonomia da pessoa em sofrimento mental como cidadã de direitos, livre de estigma, preconceito, abuso e exploração(31).

A Rede de Atenção Psicossocial (RAPS) e seus dispositivos sustentam a atenção às pessoas em sofrimento mental em todo território nacional, independentemente de sua complexidade no SUS, que também é o maior empregador dos enfermeiros na saúde mental ${ }^{(32-33)}$.

O trabalho em rede na saúde mental é complexo e dinâmico. O usuário da saúde mental movimenta-se dentro e fora de seu território, aciona serviços de outras redes, utiliza a atenção primária, secundária e terciária, convocando os profissionais da saúde a se reinventarem diante de um usuário que antes não circulava pelo espaço público. 0 modelo de atenção psicossocial no país, na perspectiva espaço-temporal, é jovem. São apenas 19 anos que separam o modelo de atenção psicossocial da psiquiatria manicomial excludente institucionalizada e juridicamente amparada.

O estudante de Enfermagem, no processo formativo da graduação, deve ter disciplinas curriculares obrigatórias em Enfermagem Psiquiátrica e Saúde Mental que desenvolvam competências para a abordagem nos cuidados primários em saúde mental, no modelo comunitário e psicossocial, com 
base dos pressupostos da Reforma Psiquiátrica, atuando na triagem e identificação de casos e utilizando tecnologias de cuidado que o capacitem para a atenção integral e humanizada das pessoas em sofrimento mental(18,21,24,34).

A reorganização dos projetos pedagógicos dos cursos de graduação em Enfermagem é emergente, tendo em vista que os conteúdos abordados e os campos de prática devem preparar o estudante de Enfermagem para o desenvolvimento da empatia, da comunicação terapêutica e do relacionamento interpessoal com a equipe, usuário e família dentro do território nos diversos pontos assistenciais que compõem a RAPS.

Porém, a literatura aponta a dificuldade de formar profissionais para a atenção psicossocial. As instituições de ensino adotam, em seus projetos pedagógicos, uma carga horária reduzida para as disciplinas de Enfermagem Psiquiátrica e Saúde Mental. Em muitas, há a ausência de integração dos conteúdos de saúde mental, como tema transversal, às outras disciplinas. Além da oferta de disciplinas que não dialogam com a realidade das pessoas em sofrimento psíquico assistidas na RAPS, há a presença de docentes sem formação na área e a realização de prática e/ou estágio desenvolvido em hospitais psiquiátricos ou serviços que mantêm a lógica manicomial de atenção, impedindo ou dificultando uma formação de qualidade em saúde mental na graduação. A literatura sugere que é improvável que os cursos de Enfermagem desenvolvam competências e habilidades necessárias ao enfermeiro para o cuidado primário em saúde mental, dada a atenção limitada à Enfermagem Psiquiátrica e Saúde Mental nesses programas(18,35-38).

Um estudo sobre a avaliação da qualidade do ensino em saúde mental por estudantes de Enfermagem apontou que os docentes deveriam ter formação na área. Quando os professores são profissionais generalistas ou especialistas em outras áreas, o ensino é frágil e o estudante, ao final do curso, não se sente preparado para atuar minimamente nos cuidados primários em saúde mental(39).

As experiências de ensino-aprendizagem em serviços mal estruturados, onde os enfermeiros não estão capacitados para a atenção em saúde mental e adotam posturas autoritárias condizentes com o modelo manicomial, sinalizam, aos estudantes, que o enfermeiro, nesse contexto, não necessita de formação específica. Os estudantes afastam-se da especialidade por não perceberem as competências necessárias para o trabalho de Enfermagem em saúde mental. Hildegard Peplau afirmava que conhecer e compreender a profissão e o seu fazer é um pressuposto para o cuidado do outro e consequente emancipação(40-41).

Em relação às atividades práticas, aponta-se a necessidade de serem desenvolvidas em serviços de saúde mental da RAPS e/ou na atenção básica com supervisão adequada e contato com profissionais capacitados para o cuidado em saúde mental primária. Nos serviços de saúde mental, a equipe de Enfermagem deve ter capacitação em Enfermagem Psiquiátrica ou Atenção Psicossocial(42).

O impacto da prática em campos de estágios mal estruturados, em especial, em hospitais psiquiátricos, e a observação do cuidado aos usuários da saúde mental, conduzida por profissionais sem qualificação para a prática, com supervisão de professores não especialistas, refletem-se no déficit de enfermeiros psiquiátricos em alguns países. Uma minoria de estudantes sente-se atraída pela especialidade por entender que pode ajudar de alguma forma o usuário da saúde mental para "salvá-lo" de uma Enfermagem inadequada e despreparada para o cuidado. Outro ponto importante é a percepção de que a atuação do enfermeiro psiquiátrico se atém a vigiar e punir os pacientes, cerceando direitos e impondo regras de comportamento que não condizem com o modelo psicossocial de atenção(43-45).

Metodologias de ensino para a Enfermagem em saúde mental têm sido aplicadas em diferentes países, tais como a rotação posicional nos serviços, o contato com o usuário de saúde mental precoce, por meio do compartilhamento de histórias e experiências dentro e fora dos serviços, o uso de simulação de atendimentos e aplicativos de anamnese em tempo real, o ensino pelo registro e a tomada de decisão considerando algoritmos comportamentais. Há especial investimento na experiência dos estudantes ouvirem e envolverem-se com histórias de vidas dos usuários da saúde mental ${ }^{(36,39,46)}$.

Alguns pontos são defendidos como imprescindíveis para a formação, dentre eles: o conhecimento das políticas públicas nacionais e locais para a área; o estudo dos determinantes e condicionantes sociais em saúde mental; a questão do financiamento das redes de atenção; o ensino de tecnologias de cuidado, com especial enfoque para os cuidados primários; a sistematização da assistência; a Enfermagem baseada em evidências; as boas práticas em Enfermagem em Saúde Mental e cenários de prática de qualidade para o treinamento discente ${ }^{(47)}$.

Estudos apontam que, nos dispositivos de atenção da RAPS, o cuidado integral em Enfermagem apresenta fragilidade, embora a Resolução no 0599/2018(42) do COFEN tenha minimizado essa questão, com especial atenção ao fazer cotidiano do enfermeiro e à definição de seu espaço e papel na equipe.

$O$ enfermeiro assume atividades que sustentam o seu fazer relacionadas à gestão da equipe de Enfermagem, mas, ao mesmo tempo, se distancia do atendimento ao usuário. Para o enfermeiro em formação, um dos possíveis direcionamentos para potencializar o ensino da Enfermagem em saúde mental é a presença de docente especialista na área, com capacidade para realizar as leituras e avaliações necessárias do contexto no qual o aluno se insere, para facilitar a construção do conhecimento de modo reflexivo e crítico. Adicionalmente, a integração ensino-serviço, com a construção dos conteúdos de forma 
articulada com as tecnologias utilizadas na RAPS, e os enfermeiros que atuam nesses possibilitam, ao estudante, pensar e refletir sobre a prática, formando profissionais comprometidos para que o cuidado de Enfermagem qualificado seja um direito de todos(48-49).

\section{O cuidado de Enfermagem em saúde mental considera a} singularidade do sujeito

A integralidade humana e as relações de cuidado na dimensão da saúde mental, incluindo a interdisciplinaridade, estão contempladas na Resolução no 573, de 31 de janeiro de 2018(17), que reconhece a comunicação e o acolhimento como tecnologias do processo de trabalho da Enfermagem com diferentes grupos sociais nos ciclos da vida.

O cuidado em saúde mental implica vínculo e corresponsabilização. Para estabelecer o vínculo, o enfermeiro utiliza tecnologias de cuidado, entre elas, a comunicação e o acolhimento, ambos citados na resolução. 0 acolhimento, o relacionamento interpessoal e a comunicação terapêutica estão relacionados ao campo teórico-prático da saúde mental, embora sejam ferramentas que podem e devem ser utilizadas em outras clínicas, com bons resultados, quando aplicadas corretamente(50-51).

As relações de cuidado pertencem ao campo das tecnologias leves do cuidar, são sensíveis aos processos de interação com o outro, são processuais e constroem-se no cotidiano do trabalho em saúde. Estudos apontam que estudantes que aprendem e aplicam essas ferramentas tendem a perceber que a saúde mental é uma área interdisciplinar que perpassa a saúde como um todo e passam a utilizar as técnicas apreendidas em outras áreas de atuação do enfermeiro(35,52-53).

Diluir os conteúdos de Enfermagem Psiquiátrica e Saúde Mental de forma transversal no currículo incorreu em fragilidades em diferentes experiências no Brasil. A transversalidade pode ser prejudicial a partir do momento em que o estudante não consegue localizar a saúde mental como uma área, quando a carga horária destinada ao tema em outras disciplinas é irrisória ou os docentes especialistas em outras áreas passam a assumir o ensino dos conteúdos específicos da Enfermagem Psiquiátrica(18,21,24,54-55).

A OMS identificou os transtornos mentais como ocupando a quinta posição em um ranking de dez causas de incapacidade, o que totaliza $12 \%$ da carga global de doenças com aumento progressivo, gerando custos sociais e econômicos. O enfermeiro tem uma posição privilegiada no campo da saúde, pois as relações de cuidado se estabelecem a partir do vínculo, o que demanda tempo e qualidade da interação. Em diferentes países, estudos apontam que o tempo dispensado ao cuidado com as pessoas pelo enfermeiro é maior do que o de outros profissionais da área da saúde ${ }^{(23,28,56-57)}$.
As relações de cuidado dão-se de forma individual e coletiva, respeitando a singularidade individual e coletiva de grupos específicos, quer seja no âmbito dos serviços de saúde, quer seja na família, comunidade e sociedade. Nesse sentido, é importante que a Resolução no 573(17) tenha explicitado, em seu texto, um capítulo destinado aos conteúdos curriculares e projetos pedagógicos, que inclui as ciências humanas e sociais como base para a compreensão das dimensões de cuidado individual e coletivo.

As ciências humanas, políticas e sociais contribuem para a compreensão crítica dos determinantes e condicionantes da saúde, como descreve o texto da resolução. Um currículo que não se preocupa com a formação humanística integral do enfermeiro aproxima-se da formação da técnica pela técnica e pouco agrega à formação cidadã do futuro profissional(16).

Importante também é a referência à pessoa com transtorno mental como sujeito do cuidado em Enfermagem. São avanços necessários e que podem contribuir para corrigir distorções em relação à carga horária destinada para as disciplinas da área da saúde mental nos currículos de Enfermagem no Brasil(38,56,58-59).

A fragilidade na formação é uma das responsáveis pela lógica do encaminhamento e da não escuta da demanda do usuário da saúde mental; outro ponto é o estigma e o preconceito. Em relação ao estigma, especialmente, o transtorno mental é associado ao comportamento imprevisível, à violência e agressividade e à dificuldade de manejo fora da clínica da Psiquiatria. As pessoas são classificadas como pacientes pertencentes à Psiquiatria. O sujeito passa a ocupar o lugar de objeto por pertencer a determinada clínica e profissionais. 0 cuidado de Enfermagem é amplo e integral e a formação deve reafirmar a singularidade da pessoa, direcionando a sua demanda em saúde de forma consciente e responsável(35,60-61).

Os conceitos de estigma e o preconceito devem ser trabalhados com os alunos, sensibilizando-os para a vulnerabilidade da pessoa em sofrimento mental e suas demandas, que vão além de delírios e alucinações. O estigma em relação à pessoa em sofrimento mental o desqualifica enquanto sujeito de direitos e justifica práticas manicomiais, assim como fomenta a defesa da exclusão e o internamento no manicômio. Para além dessa questão, o estigma dificulta o diagnóstico das pessoas em sofrimento mental nos serviços de saúde(35,62).

A tecnologia para diagnósticos e tratamento e o domínio das técnicas de intervenção são imprescindíveis no campo da saúde. Nas relações de cuidado, em especial, na saúde mental, os encontros entre o profissional de saúde e o usuário da saúde são intermediados pelo uso do acolhimento como uma tecnologia em saúde. Quando aliado à comunicação, o acolhimento pode dar visibilidade às demandas dos usuários ${ }^{(32,53)}$. 
Estudos apontam que o Centro de Atenção Psicossocial (CAPS) passa a ser o único local possível para a atenção em saúde para essa população. O CAPS é o serviço ordenador da Rede de Atenção Psicossocial e pode ser confundido com um centro de especialidades e pronto atendimento para qualquer demanda do usuário da saúde mental quando não se tem clareza de que o usuário da saúde mental tem direito ao cuidado integral(39,63).

As práticas e/ou estágios de saúde mental podem incluir, em seus campos, os serviços comunitários da RAPS, como leitos psiquiátricos em hospitais gerais, enfermarias psiquiátricas que tenham articulação com o território, CAPS, Centro de Convivência (CECCO) e Estratégia Saúde da Família (ESF). Ressalta-se que, apesar de a lei da Reforma Psiquiátrica ter quase duas décadas, a existência de hospitais psiquiátricos sinaliza que o modelo manicomial ainda é um desafio a ser superado. Nesse sentido, existem instituições de ensino que executam suas práticas naqueles espaços, o que, por conseguinte, fragiliza a formação do enfermeiro dentro do modelo psicossocial ${ }^{(18)}$. Além disso, é preciso sinalizar que podem existir atitudes e cuidado exercidos por profissionais de saúde na RAPS no modelo manicomial e isso requer atenção e elaboração de indicadores de qualidade por parte dos docentes para a seleção e a articulação, juntamente ao município, dos serviços de saúde que podem ser campos de prática.

Uma investigação em 89 instituições de ensino públicas no Brasil desvelou que somente (24) 23,3\% dos docentes conduzem o ensino de saúde mental apenas na atenção primária à saúde com estratégias de visita domiciliar, ações educativas e busca ativa de casos de saúde mental. Essa realidade revela que o ensino de saúde mental ainda está centrado nos cenários de especialidade, o que dificulta que o processo formativo do enfermeiro generalista contemple competências para que este consiga prover cuidados primários em saúde mental, identificar precocemente sujeitos em sofrimento psíquico e atuar no tratamento de quadros psiquiátricos estáveis, na prevenção e promoção da saúde mental(64).

Para atender às questões contemporâneas e fortalecer a Enfermagem como campo de saber autônomo e comprometido com os pressupostos da Reforma Psiquiátrica, a Associação Brasileira de Enfermagem, com a criação do Departamento de Saúde Mental, buscou fortalecer politicamente a Enfermagem Psiquiátrica em defesa da reforma psiquiátrica, da desinstitucionalização dos saberes e práticas associados à lógica manicomial, posicionando a Enfermagem brasileira em relação à defesa dos direitos dessa população a um tratamento digno e de qualidade, dando visibilidade para a área(59).

Ressignificar a loucura de forma política, social e cultural é uma estratégia que pode ser utilizada para deslocar a formação dos estudantes de Enfermagem na graduação do modelo assistencial institucionalizante e excludente e ter como instrumentalização a relação terapêutica e a qualificação do cuidado em saúde mental pelo enfermeiro.

\section{Considerações finais}

As recomendações para a reformulação das DCNs do curso de graduação em Enfermagem sinalizam alterações necessárias e arrojadas para atender às demandas sociais, políticas e de mercado na contemporaneidade. Para a Enfermagem, tais reformulações apresentam avanços importantes na formação do enfermeiro generalista.

Entende-se que cabe aos enfermeiros docentes, como educadores à frente do processo de formação, discutirem o impacto na avaliação e reformulação dos projetos políticos pedagógicos e currículos das escolas de Enfermagem em todo o país.

A base para o desenvolvimento de uma profissão na área da saúde é a formação de qualidade, implicada com o bem-estar social e a qualidade de vida de indivíduos e populações. A Enfermagem Psiquiátrica e em Saúde Mental, diante dos dados epidemiológicos e das projeções de sofrimento mental para a população mundial, será instada a assumir cuidados gerais e específicos de saúde mental, expandindo uma área do conhecimento da Enfermagem que pouco tem atraído os futuros enfermeiros. As mudanças propostas no campo formador atendem às necessidades que se apresentam agora e no futuro.

Aponta-se a necessidade da realização de estudos que identifiquem o adoecimento mental como algo intrinsecamente ligado aos condicionantes e determinantes sociais de saúde e saúde mental, bem como no campo do cuidado primário em saúde mental no ensino da graduação, identificando as tecnologias do cuidado e as competências necessárias para o enfermeiro generalista atuar na atenção à pessoa em sofrimento mental em diferentes cenários.

\section{Referências}

1. El-Hage $W$, Hingray $C$, Lemogne $C$, Yrondi A, Brunault $P$, Bienvenu $T$, et al. Les professionnels de santé face à la pandémie de la maladie à coronavirus (COVID-19): quels risques pour leur santé mentale? Enchephale. 2020 Jun;46:S73-S80. doi: http://doi.org/10.1016/j. encep.2020.04.008

2. Chevance A, Gourio D, Hoerte N, Llorca P, Thomas P, Bocher $R$, et al. Ensuring mental health care during the SARS-CoV-2 epidemic in France: A narrative review. Encephale. 2020 Jun;46:193-201. doi: http://doi. org/10.1016/j.encep.2020.04.005

3. Zhenyu L, Jingwu G, Meiling $Y$, Jianping $F$, Mei Q, Riyue $\mathrm{J}$, et al. Vicarious traumatization in the general public, members, and non-members of medical teams aiding in 
COVID-19 control. Brain Behav Immunol. 2020 Aug;88:9169. doi: http://doi.org/10.1016/j.bbi.2020.03.007

4. Silva DAR, Pimentel RFW, Merces MC. Covid-19 and the pandemic of fear: reflections on mental health. Rev Saúde Pública. 2020 May;54:46. doi: http://doi.org/10.11606/ s1518-8787.2020054002486

5. All-Party Parliamentary Group on Global Health (APPG). Triple Impact - how developing nursing will improve health, promote gender equality and support economic growth [Internet]. London (UK): APPG; 2016 [cited 2020 Sep 3]. Available from: https://www.who.int/hrh/com-heeg/digitalAPPG_triple-impact.pdf?ua $=1$

6. Cassiani SHB, Lira JCG Neto. Nursing Perspectives and the "Nursing Now" Campaign. Rev Bras Enferm. 2018 Oct;71(5):2351-2. doi: http://doi. org/10.1590/0034-7167.2018710501

7. McEnroe N. The art of medicine Celebrating Florence Nightingale's bicentenary. Lancet. 2020 May;395(10235):1475-8. doi: http://doi.org/10.1016/ S0140-6736(20)30992-2

8. Ximenes FRG Neto, Lopes D Neto, Cunha ICKO, Ribeiro MA, Freire NP, Kalinowski CE, et al. Reflections on Brazilian Nursing Education from the regulation of the Unified Health System. Ciênc Saúde Coletiva. 2020 Jan;25(1):37-46. doi: http://doi.org/10.1590/1413-81232020251.27702019

9. Pepin JI, Myrick F. A conversation on nursing education research with the 2018 Pat Griffin Scholar, Dr Linda Ferguson. Qual Adv Nurs Educ. 2019 Apr;5(1):1-13. doi: http://doi.org/10.17483/2368-6669.1211

10. Pan American Health Organization. Strategic Directions for Nursing in the Region of the Americas [Internet]. Washington: PAHO; 2019 [cited 2020 Sep 3]. Available from: http://iris.paho.org/xmlui/ handle/123456789/50956?locale-attribute $=$ en

11. Machado MH, Frota MA, Wermelinger MCMW, Ximenes FRG Neto, Freire NP. Health and Nursing Systems: the national and international context. Ciênc Saúde Coletiva. 2020 Jan;25(1):4. doi: http://doi. org/10.1590/1413-81232020251.28562019

12. Teixeira E, Dumêt JC, Andrade AC, Silva KL, Rocha MEMO, Lima RJO. Overview of Nursing Graduation courses in Brazil in the National Curriculum Guidelines decade. Rev Bras Enferm. 2013 Set;66:102-10. doi: ttp://doi.org/h10.1590/ S0034-71672013000700014

13. Conselho Nacional de Educação (BR). Câmara de Educação Superior. Resolução CNE/CES n० 3 de 7 de novembro de 2001. Institui Diretrizes Curriculares Nacionais do Curso de Graduação em Enfermagem. Brasília: CNE/ CES; 2001.

14. Costa DAS, Silva RF, Lima VV, Ribeiro ECO. National curriculum guidelines for health professions 2001-2004: an analysis according to curriculum development theories. Interface. (Botucatu). 2018 Ago;22(67):1183-95. doi: http://doi.org/10.1590/1807-57622017.0376
15. Adamy EK, Teixeira E. The quality of education in times of new National Curriculum Parameters. Rev Bras Enferm. 2018;71(Suppl 4):1485-6. doi: http://doi. org/10.1590/0034-7167-201871sup401

16. Nascimento MC, Guedes $C R$, organizadores. Saúde, Sociedade e Cultura: Ciências Sociais e Humanas para a Graduação em Saúde. São Paulo: Editora Hucitec; 2017. $232 \mathrm{p}$.

17. Ministério da Saúde (BR). Conselho Nacional de Saúde. Resolução no 573 de 31 de janeiro de 2018 (BR). Recomendações do Conselho Nacional de Saúde à proposta de Diretrizes Curriculares Nacionais (DCN) do curso de graduação Bacharelado em Enfermagem. [Internet]. Brasília: Diário Oficial da União, 6 nov 2018 [Acesso 19 mai 2021]. Disponível em: https://www.in.gov.br/materia/-/ asset_publisher/Kujrw0TZC2Mb/content/id/48743098/ do1-2018-11-06-resolucao-n-573-de-31-de

18. Olmos CEF, Rodrigues J, Lino MM, Fernandes JD, Lazzari DD. Psychiatric nursing and mental health teaching in relation to Brazilian curriculum. Rev Bras Enferm. 2020 Mar;73(2):e20180200. doi: http://doi. org/10.1590/0034-7167-2018-0200

19. Frota MA, Wermelinger MCMW, Vieira LJES, Ximenes FRG Neto, Queiroz RSM, Amorim RF. Mapping nursing training in Brazil: challenges for actions in complex and globalized scenarios. Ciênc Saúde Coletiva. 2020 Jan;25(1):25-35. doi: http://doi.org/10.1590/1413-81232020251.27672019 20. Bezerra IC, Jorge MSB, Gondim APS, Lima LL, Vasconcelos MGF. "I went to the health unit and the doctor sent me here": process of medicationalization and (non)resolution of mental healthcare within primary care". Interface. (Botucatu). 2014;18(48):61-74. doi: http://doi. org/10.1590/1807-57622013.0650

21. Siqueira AC Júnior, Otani MAP. The teaching of psychiatric nursing and mental health in the curriculum by competence. REME - Rev Min Enferm. [Internet]. 2011 Out/Dez [cited 2020 Set 3];15(4):539-45. Available from: http://reme.org.br/artigo/detalhes/68

22. Mattia BJ, Kleba ME, Prado ML. Nursing training and professional practice: an integrative review of literature. Rev Bras Enferm. 2018;71(4):2039-49. doi: http://doi. org/10.1590/0034-7167-2016-0504

23. Cantante APSR, Fernandes HIVM, Teixeira MJ, Frota MA, Rolim KMC, Albuquerque FHS. Health Systems and Nursing Skills in Portugal. Ciênc Saúde Coletiva. 2020 Jan;25(1):261-72. doi: http://doi. org/10.1590/1413-81232020251.27682019

24. Tavares CMM, Gama LN, Souza MMT, Paiva LM, Silveira PG, Mattos MMGR. Specific skills of mental health nurses in undergraduate nursing teaching. Rev Portuguesa Enferm Saúde Mental. 2016 Out;Spe 4:25-32. doi: http://doi. org/10.19131/rpesm.0137

25. Rehm J, Kilian C, Ferreira Borges C, Jernigan D, Monteiro M, Parry C, et al. Alcohol use in times of the COVID 
19: Implications for monitoring and policy. Drug Alcohol Rev. 2020 Mai;39(4):301-4. doi: http://doi.org/10.1111/ dar. 13074

26. Organização Mundial de Saúde (OMS). A saúde mental pelo prisma da saúde pública. Relatório sobre a saúde no mundo 2001: Saúde mental: nova concepção, nova esperança. Genebra: OPAS/OMS; 2001. 205 p.

27. Poupart J, Deslauriers JP, Groulx LH, Laperriére A, Mayer R, Pires AP, et al. A pesquisa qualitativa: enfoques epistemológicos e metodológicos. Petrópolis: Vozes; 2008. $464 \mathrm{p}$.

28. Bardin L. Análise de conteúdo. Lisboa: Edições70; 2016. $280 \mathrm{p}$.

29. Souza FN, Costa AP, Moreira A, Souza DN, Freitas F. webQDA - Manual de Utilização Rápida. Aveiro: UA Editora Universidade de Aveiro Serviços de Biblioteca, Informação Documental e Museologia; 2016. 30 p.

30. Conselho Nacional de Educação (BR). Resolução CNE/ CES no 3 de 7 de novembro de 2001. Institui Diretrizes Curriculares Nacionais do Curso de Graduação em Enfermagem. Brasília (DF): Diário Oficial da União, 9 nov 2001.

31. Brasil. Lei no 10.216 de 6 de abril de 2001. Dispõe sobre a proteção e os direitos das pessoas portadoras de transtornos mentais e redireciona o modelo assistencial em saúde mental. Brasília (DF): Diário Oficial da União, 9 abr 2001.

32. Ministério da Saúde (BR). Portaria n 3.088 de 23 de dezembro de 2011. Institui a Rede de Atenção Psicossocial para pessoas com sofrimento ou transtorno mental e com necessidades decorrentes do uso de crack, álcool e outras drogas, no âmbito do Sistema Único de Saúde (SUS). Brasília (DF): Diário Oficial da União, 30 dez 2011.

33. Jorge MSB, Pinto DM, Quinderé PHD, Pinto AGA, Sousa FSP, Cavalcante CM. Promotion of Mental Health Technologies for Care: emotional involvement, reception, co-responsibility and autonomy. Ciênc Saúde Coletiva. 2011 Jul;16(7):3051-60. doi: http://doi.org/10.1590/ S1413-81232011000800005

34. Machado $\mathrm{MH}$, coordenador. Perfil da enfermagem no Brasil: relatório final. Rio de Janeiro (RJ): NERHUS - DAPS - ENSP/Fiocruz; 2017. 748 p.

35. Çingöl N, Karakas M, Zengin S, Çelebi E. The effect of psychiatric nursing students' internships on their beliefs about and attitudes toward mental health problems; a single-group experimental study. Nurse Educ Today. 2020 Jan;84(104243):1-7. doi: http://doi.org/10.1016/j. nedt.2019.104243

36. Happell B, Waks S, Bocking J, Horgan A, Manning F, Greaney $S$, et al. 'There's more to a person than what's in front of you': Nursing students' experiences of consumer taught mental health education. Int J Ment Health Nurs. 2019 Ago;28(4):950-9 doi: http://doi.org/10.1111/ inm.12596
37. Cassiani SHB, Jimenez EFM, Ferreira AU, Peduzzi M, Hernández CL. La situación de la enfermería en el mundo y la Región de las Américas en tiempos de la pandemia de COVID-19. Rev Panam Salud Publica. 2020 Mai;44:e64. doi: http://doi.org/26633/RPSP.2020.64

38. Pereira MA, Reinaldo MAS, Villa EA, Gonçalves $A M$. Overcoming the challenges to offer quality training in psychiatric nursing. Rev Bras Enferm. 2020 Fev;73(1):e20180208. doi: http://doi. org/10.1590/0034-7167-2018-0208

39. Campbell K, Massey D, Broadbent M, Clarke KA. Factors influencing clinical decision making used by mental health nurses to provide provisional diagnosis: A scoping review. Int J Ment Health Nurs. 2019 Abr;28(2):407-24. doi: http:// doi.org/10.1111/inm.12553

40. George JB. Teorias de enfermagem: os fundamentos para a prática profissional. Porto Alegre (RS): Artes Médicas Sul; 2000. 235 p.

41. Slemon A, Jenkins $E$, Bungay $V$, Brown $H$. Undergraduate students' perspectives on pursuing a career in mental health nursing following practicum experience. J Clin Nurs. 2020 Jan;29(1-2):163-71. doi: http://doi.org/10.1111/ jocn. 15074

42. Brasil. Conselho Federal de Enfermagem. Resolução COFEN no 599 de 19 de dezembro de 2018. Aprova Norma Técnica para Atuação da Equipe de Enfermagem em Saúde Mental e Psiquiatria. Brasília (DF): COFEN; 2018.

43. Rice MJ, Stalling J, Monasterio A. PsychiatricMental Health Nursing: Data Driven Policy Platform for a Psychiatric Mental Health Care Workforce. J Am Psychiatr Nurses Assoc. 2019 Jan-Fev;25(1):27-37. doi: http://doi. org/10.1177/1078390318808368

44. Stuhlmillera C, Tolchard B. Understanding the impact of mental health placements on student nurses' attitudes towards mental illness. Nurse Educ Practice. 2019 Jan;34:2530. doi: http://doi.org/10.1016/j.nepr.2018.06.004 45. Franzese J, Pecinka K, Schwenk J. Alternative Clinical Experience Through Academic Service-Learning Develops Into a Partnership for Mental Health Rotation. Teach Learn Nurs. 2020 Jan;15(1):77-81. doi: http://doi.org/10.1016/j. teln.2019.10.002

46. Bourla A, Mouchabac S, Ogorzelec L, Guinchard C, Ferreri F. Are student nurses ready for new technologies in mental health? Mixed-methods study. Nurse Educ Today. 2020 Jan;84(104240):1-7. doi: http://doi.org/10.1016/j. nedt. 2019.104240

47. Cioffi ACS, Ribeiro MRR, Ormonde JC Júnior. Validation of the competence profile proposal for the training of nurses. Texto Contexto Enferm. 2019 Mar;28(e20170384):1-15. doi: http://doi.org/10.1590/1980-265x-tce-2017-0384

48. Hirdes A, Scaparo HBK. The maze and the minotaur: mental health in primary health care. Ciênc Saúde Coletiva. 2015 Fev;20(2):383-93. doi: http://doi. org/10.1590/1413-81232015202.12642013 
49. Cardoso MRO, Oliveira PTR, Piani PPF. Care practices in mental health in the voice of users from a Psychosocial Care Center of the state of Pará. Saúde Debate. 2016;40(109):8699. doi: http://doi.org/10.1590/0103-1104201610907

50. Telles LL, Jardim SR, Rotenberg L. Call me for a conversation and I will enjoy it: analysis of a clinicalinstitutional experience with the nursing staff of a psychiatric hospital. Ciênc Saúde Coletiva. 2020 Jan;25(1):181-90. doi: http://doi.org/10.1590/1413-81232020251.28882019 51. Frosi RV, Tesser CD. Mental health care practices in primary health care: an analysis based on experiences developed in Florianópolis, Brazil. Ciênc Saúde Coletiva. 2015;20(10):3151-61. doi: http://doi. org10.1590/1413-812320152010.10292014

52. Merhy EE. Saúde: Cartografia do Trabalho vivo. São Paulo: Hucitec; 2014. 192 p.

53. Campos DB, Bezerra IC, Jorge MSB. Mental health care technologies: Primary Care practices and processes. Rev Bras Enferm. 2018;71(Suppl 5):2101-8. doi: http://doi. org/10.1590/0034-7167-2017-0478

54. Rodrigues J, Santos SMA, Zeferino MT, Tosoli M. Integrative review about the teaching of the nursing care in mental health. J Res Fundam Care Online. 2014 Jan-Mar; 6(1):433-49. doi: http://doi.org/10.9789/2175-5361.2014. v6i1.433-449

55. Onocko-Campos RT. Mental health in Brazil: strides, setbacks, and challenges. Cad Saude Pública. 2019 Out;35(11):e00156119. doi: http://doi. org/10.1590/0102-311x00156119

56. Pan American Health Organization. Expanding the Roles of Nurses in Primary Health Care. Washington (DC): PAHO; 2018. 54 p.

57. Organização Mundial de Saúde, Organização Mundial de Médicos de Família (Wonca). Integração da saúde mental nos cuidados de saúde primários: uma perspectiva global. Genebra: OMS/Wonca; 2008. 250 p.

58. Cassiani SHB, Silva FAM. Expanding the role of nurses in primary health care: the case of Brazil. Rev. Latino-Am. Enferm. 2019 Dez;27(e3245):1-3. doi: http://doi. org/10.1590/1518-8345.0000.3245

59. Esperidião E, Silva NS, Caixeta CC, Rodrigues J. The Psychiatric Nursing, ABEn and the Scientific Department of Psychiatric and Mental Health Nursing: progress and challenges. Rev Bras Enferm. 2013 Set;66(n. spe):171-6. doi: http://doi.org/10.1590/S0034-71672013000700022 60. Inan FS, Günüşen N, Duman ZÇ, Ertem MY. The Impact of Mental Health Nursing Module, Clinical Practice and an Anti-Stigma Program on Nursing Students' Attitudes toward
Mental Illness: A Quasi-Experimental Study. J Prof Nurs. 2019 Mai-Jun;35(3):201-8. doi: http://doi.org/10.1016/j. profnurs.2018.10.001

61. Nascimento LA, Leão A. Social stigma and internalized stigma: the voice of persons with mental disorders and the confrontations required. Hist Cienc Saúde-Manguinhos. 2019 Jan-Mar;26(1):103-21. doi: http://doi.org10.1590/ S0104-59702019000100007

62. Schomerus G, Stolzenburg S, Freitag S, Speerforck $S$, Janowitz D, Evans-Lacko $S$, et al. Stigma as a barrier to recognizing personal mental illness and seeking help: a prospective study among untreated persons with mental illness. Eur Arch Psychiatry Clin Neurosci. 2019 Jun;269(4):469-79. doi: http://doi.org/10.1007/ s00406-018-0896-0

63. Frateschi MS, Cardoso CL. Mental Health in Primary Care: evaluation from the viewpoint of users. Physis. 2014;24(2):545-65. doi: http://doi.org/10.1590/ s0103-73312014000200012

64. Nóbrega MPSS, Venzel CMM, Sales ES, Próspero AC. Mental health nursing education in Brazil: perspectives for primary health care. Texto Contexto Enferm. 2020 Fev;29(e20180441):1-13. doi: http://doi. org/10.1590/1980-265x-tce-2018-0441

\section{Contribuição dos autores}

Concepção e desenho da pesquisa: Amanda Márcia dos Santos Reinaldo. Obtenção de dados: Amanda Márcia dos Santos Reinaldo, Girliani Silva de Sousa, Belisa Vieira da Silveira. Análise e interpretação dos dados: Amanda Márcia dos Santos Reinaldo, Girliani Silva de Sousa, Belisa Vieira da Silveira. Redação do manuscrito: Amanda Márcia dos Santos Reinaldo, Girliani Silva de Sousa, Belisa Vieira da Silveira. Revisão crítica do manuscrito quanto ao conteúdo intelectual importante: Amanda Márcia dos Santos Reinaldo, Girliani Silva de Sousa, Belisa Vieira da Silveira.

Todos os autores aprovaram a versão final do texto.

Conflito de interesse: os autores declararam que não há conflito de interesse. 